\title{
EFFECTIVENESS OF CONVENTIONAL VS. HYPO FRACTIONATED RT SCHEDULES FOR CHEST WALL IRRADIATION IN BREAST CANCER TREATMENT AND OUTCOME
}

\author{
Suresh Kumar K1, Kiran P2, Joneetha Jones ${ }^{3}$, Mintu Mathew ${ }^{4}$, Snigdha P. P5
}

${ }_{1}^{1}$ Additional Professor, Department of Radiotherapy and Oncology, Cancer Care Center Medical College, Kottayam, Kerala, India. ${ }^{2}$ Assistant Professor, Department of Radiotherapy and Oncology, Cancer Care Center Medical College, Kottayam, Kerala, India. 3 Senior Resident, Department of Radiotherapy and Oncology, Cancer Care Center Medical College, Kottayam, Kerala, India. ${ }^{4}$ Senior Resident, Department of Radiotherapy and Oncology, Cancer Care Center Medical College, Kottayam, Kerala, India. ${ }_{5}^{5}$ Senior Resident, Department of Radiotherapy and Oncology, Cancer Care Center Medical College, Kottayam, Kerala, India.

\section{ABSTRACT}

\section{BACKGROUND}

Post mastectomy radiotherapy for patients with positive nodes derives a disease-free survival and overall survival benefit. Radiobiological models suggest that hypo fractionation might be equally effective for adjuvant RT in breast cancer. Evidence has been convincing, and this is now being accepted as standard practice.

\section{MATERIALS AND METHODS}

This retrospective cohort study compares the two fractionation schedules in Carcinoma Breast used in post mastectomy chest wall irradiation ( $50 \mathrm{~Gy} / 25$ fractions vs $40 \mathrm{~Gy} / 15$ fractions) regarding loco-regional control and toxicity profile. Patients were accrued from March 2011 to August 2013 and were followed up for a period of 1 year. A total of 195 patients were included in the study.

\section{RESULTS}

The baseline characteristics were similar in both arms. No statistically significant difference in disease free survival was seen between the two arms. The toxicity profile was also comparable.

\section{CONCLUSION}

After mastectomy for breast cancer, a radiotherapy schedule delivering 40 Gy in 15 fractions over 3 weeks seems to offer local regional tumour control and rates of normal tissue effects are at least as good as the accepted international standard of 50 Gy in 25 fractions over 5 weeks

\section{KEY WORDS}

Breast Cancer, Radiation, Hypofractionation.

HOW TO CITE THIS ARTICLE: Kumar SK, Kiran P, Jones J, et al. Effectiveness of conventional vs. hypo fractionated RT schedules for chest wall irradiation in breast cancer treatment and outcome. J. Evolution Med. Dent. Sci. 2019;8(02):104-110, DOI: $10.14260 /$ jemds/2019/23

\section{BACKGROUND}

Breast cancer treatment is a multimodality approach with Surgery, Radiation and systemic therapy. Post mastectomy radiotherapy for patients with positive nodes derives a disease-free survival and overall survival benefit. Most centers around the world treat post mastectomy chest wall and draining areas to a total dose of 50 Gy in 25 fractions delivered with conventional fractionation of 2 Gy per fraction for 5 days a week lasting for 5 weeks.

Radiobiological models suggest that a hypo fractionated regime (fractionation use a dose of more than 2 Gy per fraction with decrease in total number of fractions) might be equally effective. The more commonly employed fractionation schedule is 40 Gy in 15 fraction. ${ }^{1}$

'Financial or Other Competing Interest': None.

Submission 03-12-2018, Peer Review 28-12-2018,

Acceptance 04-01-2019, Published 14-01-2019.

Corresponding Author:

Dr. Kiran $P$,

Assistant Professor,

Department of Radiotherapy and Oncology,

Cancer Care Center Medical College,

Kottayam, Kerala,

India.

E-mail: kiranphere@gmail.com

DOI: $10.14260 /$ jemds $/ 2019 / 23$

\section{(c) (i) $(9)$}

This regimen may also be more convenient for patients and less resource intensive than the standard schedule. Low rates of local recurrence and limited or comparable radiationinduced morbidity have been reported with such approaches. ${ }^{2}$

In Department of radiotherapy at our institute we are treating approximately 3200 new patients every year, out of which 10 to $15 \%$ are having carcinoma breast. 60 to $70 \%$ among these are locally advanced disease and will receive post mastectomy chest wall irradiation. The post mastectomy patients receive radiation with conventional fractionation of 2 Gy per day for 5 days a week last for 5 weeks ( 50 Gy). This requires prolonged hospital stay resulting in increased cost of treatment, increased workload and decreased patient compliance. Hence adopting a shorter fractionation regime of 40 Gy in 15 fractions in which 2.66 Gy is delivered per day and for 5 days a week in which the entire radiation treatment would be over in 3 weeks, with equivalent loco-regional control and acceptable toxicity profile will definitely overcome the drawbacks of conventional fractionation and improve patient compliance. Consequently, we can treat a greater number of patients per day with better utilization of available resources. This study intends to compare a standard fractionation of 50 Gy in 25 fractions to a hypo fractionated regime of $40 \mathrm{~Gy}$ in 15 fractions in post mastectomy chest wall irradiation in Carcinoma Breast with a focus on toxicity profile for Indian patients. 


\section{Review of Literature \\ Epidemiology}

Breast cancer is the most frequent cancer among women with an estimated 2.46 million new cancer cases diagnosed in 2017 (15.3\% of all cancers).It is the most common cancer both in developed and developing regions with around 883000 new cases estimated in each region. 3 The range of mortality rates is much less (Approximately 6-20 per 100, 000) because of the more favourable survival of breast cancer in developed regions. ${ }^{3}$ In India it ranks the second commonest cancer in incidence and is on the rise. ${ }^{4}$

Carcinoma Breast has been divided into early stage, locally advanced and Metastatic breast cancer and uses a multimodality approach. Overall survival of Breast cancer patients correlates with the stage of disease at presentation. For early stage disease the recommended treatment is surgical followed by adjuvant radiotherapy and chemotherapy. Neo adjuvant chemotherapy followed by surgery is used in locally advanced tumours which is followed by adjuvant systemic treatment and radiotherapy according to clinico-pathological findings. ${ }^{5}$

The surgical management of patients address both the primary tumour and regional lymphatics. The primary tumour may be managed by mastectomy or lumpectomy, and the nodal regions may be surgically addressed by lymph node dissection or sentinel node biopsy. Level 1 and 2 axillary dissection required that at least 10 nodes should be provided to pathologically stage the axilla. ${ }^{5}$

\section{Radiotherapy}

The worldwide trend to be less radical treatment in the management of Carcinoma Breast has led to increasing role of radiotherapy in this disease. As the risk of local recurrence after mastectomy was 10 to $15 \%$ it was hoped that prophylactic radiotherapy would decrease this. Early Breast Cancer Trialists' Collaborative Group and 2002 Cochrane review $^{6}$ have shown that postoperative radiotherapy decreases recurrence rates by two third. Post-operative RT decreases recurrence rates in stage 1 by $5 \%$, stage 2 by $10 \%$ and stage 3 by 10 to $15 \% .^{6}$

The technique of breast irradiation has evolved from use of conventional two-dimensional planning with and without using wedges to $3 \mathrm{D}$ conformal radiotherapy and intensity modulated radiotherapy (IMRT). Standard opposed tangential fields with appropriate use of wedges to optimize dose homogeneity remain the most commonly employed method for delivery of whole breast irradiation. For some women, the 6-week course of daily radiation with its associated time and travel issues is not feasible. In response to this, a wide variety of accelerated forms of treatment have been developed and proven safe and effective in short-term studies.

\section{Post Mastectomy Radiotherapy}

Defined as delivery of adjuvant RT to chest wall +/- lymph nodes. Post mastectomy irradiation is indicated in patients with T4 tumours, primary tumour larger than $5 \mathrm{~cm}$ (T3), and involvement of axillary nodes and close or positive pathologic margins. The findings of the $2005 \mathrm{EBCTG}^{7}$ meta-analysis proved the need for post mastectomy radiation in all nodepositive patients. National Comprehensive Cancer Network
(NCCN) also recommends to strongly consider RT in 1-3 node positive patients. ${ }^{5}$

\section{Volumes and Techniques}

The areas treated generally include the chest wall and supraclavicular, axillary regions.

\section{Chest Wall Irradiation}

The entire ipsilateral chest wall should be encompassed. Conventional field borders are determined clinically using the mastectomy scar and anatomic landmarks from the contralateral breast. Chest wall is treated using two tangential fields, medial and lateral tangents. Superior border is kept at second intercostal space. Medial border is at midline or matched with internal mammary field when used. Lateral border is kept at midaxillary line. Inferior border is at $2 \mathrm{cms}$. below opposite inframammary fold. ${ }^{8}$

\section{Supraclavicular Field}

Supraclavicular nodes are usually encompassed, with a single anterior oblique photon field angled slightly away from the spinal cord. The medial border is placed at the insertion of the clavicular head. The lateral border is placed to include approximately one-third of the humeral head. The inferior border is the inferior border of the clavicle or the superior border of the chest wall field. The superior border is set at the thyrocricoid groove. A humeral head block is added to block the humeral head and acromioclavicular joint. ${ }^{8}$

To avoid the problem of hot spot while matching supraclavicular and chest wall fields following methods are used. Angling the foot of the treatment couch away from the radiation source to direct the tangential fields inferiorly so that superior edge of these beams line up perfectly with inferior border of supraclavicular field. We can also use a three-field single isocentre technique or Hanging block technique. Half beam block can also be used. ${ }^{8}$

\section{Dose}

Most commonly employed dose schedule historically is 50 Gy in 25 fractions at conventional 2 Gy daily for 5 weeks. Hypo fractionated schedules are also being used in various trials like 40 Gy in 15 fractions/42.6 Gy in 15 fractions/39 Gy in 13 fractions.

\section{Hypofractionation}

Hypo fractionation was introduced in the United Kingdom (UK) and Canada several decades ago on an empirical basis. Results of retrospective studies of hypo fractionated radiotherapy in breast cancer suggest satisfactory outcomes in terms of tumour control and late adverse effects if modest increases in fraction size are combined with appropriate downward adjustments to total dose. 9

\section{Radiobiology}

It has been understood that as fraction size increases, total dose must be reduced in order to maintain the same level of antitumor or normal tissue effect. It has been shown by radiobiological analysis of clinical data, that breast adenocarcinomas have an $\alpha / \beta$ ratio of around $4 \mathrm{~Gy}$, i.e. close to late reacting normal tissues. Consequently, hypofractionation in breast cancer may have a reasonable radiobiological background as more tumour cells will be killed by a high dose 
per fraction compared with the conventional 2 Gy per fraction, and would potentially compensate for repopulation of tumour cells during radiation treatment (RT). ${ }^{10}$

\section{Toxicities of Chest Wall Irradiation}

Side effects or toxicities can be separated into 2 general categories: early and late. Early or acute toxicities occur during the course of radiation, whereas late toxicities may occur 6 months to several years after radiation. The most common acute side effects from radiation are fatigue and skin irritation. These side effects, and all others, vary greatly from patient to patient.

Skin irritation (radiation dermatitis) is a common. Skin toxicity is assessed using Radiation Therapy Oncology Group Criteria(RTOG) ${ }^{11}$. Other symptoms which patients may complain during radiation treatment include of difficulty in swallowing, pain or respiratory symptoms like cough and dyspnoea.

\section{Late Effects}

The most frequent long-term risks of PMRT include lymphedema, brachial plexopathy, radiation pneumonitis, rib fractures, cardiac toxicity, and radiation-induced second neoplasms.

\section{Objectives of the Study}

1. To compare the two fractionation schedules in Carcinoma Breast used in post mastectomy chest wall irradiation regarding loco-regional control.

2. To compare the toxicity profile of two radiation fractionation schedules.

\section{MATERIALS AND METHODS}

\section{Study Design}

This study is a retrospective cohort study, comparing locoregional control and toxicity profile of two fractionation schedules used in post mastectomy chest wall irradiation in Carcinoma Breast.

\section{Inclusion Criteria}

1. Female patients of Carcinoma Breast post Mastectomy, in whom radiation treatment is indicated and are attending Radiotherapy department from March 2011 to August 2013 were included in the study.

2. Female gender.

3. Histopathologically proven.

4. Post Mastectomy and Axillary dissection.

5. Early stage disease with lymph node positivity.

6. All T3, T4 cases.

7. Positive or close resection margins

8. Those who gave written informed consent.

\section{Exclusion Criteria}

1. Patients who were not willing for the study.

2. Age $>/=70$ yrs.

3. Patients with Karnofsky Performance (KPS) status less than $70 \%$.

4. Those who underwent Breast conservation surgery.

5. Metastatic Carcinoma Breast.

\section{Study Setting}

All patients of Carcinoma Breast after Mastectomy, who fulfilled the inclusion criteria and had attended Radiotherapy
Department from March 2011 to August 2013 were included in the study.

\section{Duration of the Study}

Patients were accrued from March 2011- August 2013 in this study. All patients were followed up for a period of 1 year following completion of external beam radiotherapy.

\section{Sample Size}

195 patients (96 patients in Arm A and 99 patients in Arm B) was taken for convenience.

\section{Treatment Protocol}

All patients accrued in this study were planned for external beam radiation using the Conventional fluoroscopic $\mathrm{X}$ ray simulator housed in the Department of Radiotherapy. The treatment fields were defined following $\mathrm{x}$-ray imaging of the involved region on the x-ray simulator. Chest wall was treated with two tangential fields in SSD (Source to skin distance) technique with conventional field borders. A single on field was planned for supraclavicular fossa. Posterior axillary boost was also given as per standard protocol of the institution.

Patients were selected and compared after matching for stage. Arm A patients had received 50 Gy in 25 fractions to chest wall and 50 Gy in 25 fractions to supraclavicular fossa. Arm B patients received 40 Gy in 15 fractions to chest wall and $45 \mathrm{G}$ y in 15 fractions to supraclavicular fossa. All patients received a posterior axillary boost of 6 Gy single fraction as per institutional protocol.

All patients in this study were treated with either the Theratron 780C Telecobalt therapy unit or $6 \mathrm{MV}$ Linear accelerator housed in the Department of Radiotherapy.

\section{Follow Up}

Patients were assessed after every 5 fractions for toxicity during radiation treatment and findings were recorded. Subsequently patients were followed up monthly for the first 3 months and once in two months for a period of two year from date of completion of radiotherapy.

\section{Assessment Tools}

Loco-regional control was assessed by clinical examination. The chest wall, axilla and supraclavicular fossa were examined to rule out any scar recurrence, chest wall recurrence, cutaneous nodules or the presence of any lymph nodes in axilla, internal mammary area or supraclavicular fossa. All findings were recorded.

Toxicity profile was assessed with RTOG toxicity grading. Skin and Lung toxicity was graded based on RTOG scoring. Presence or absence of odynophagia, lymphedema, subcutaneous fibrosis, brachial plexopathy, rib fracture and occurrence of second malignancies were also recorded.

\section{Statistical Analysis}

Data was entered in Microsoft Excel \& analysis was done using the Statistical package for social sciences (SPSS Inc., Chicago, IL) version 20.0 software. Statistical significance of difference in proportion was computed using chi square test or the t test, respectively. Disease free survival was plotted using Kaplan Meier. Significance was assessed with Breslow test. A p value of $<0.05$ was taken as significant. 


\section{RESULTS}

\section{Descriptive data}

195 patients who satisfied the criteria for patient selection from March 2011 to August 2013 were accrued in this study.

Baseline patient characteristics are summarized in the table. There were no significant differences in any of the major baseline characteristics among patients.

\section{Baseline Characters}

\begin{tabular}{|c|c|c|c|}
\hline & $\begin{array}{c}\text { ARM A (50 } \\
\text { Gy/25) }\end{array}$ & $\begin{array}{c}\text { ARM B (40 } \\
\text { Gy/15) }\end{array}$ & p-Value \\
\hline \multicolumn{4}{|l|}{ Age } \\
\hline $30-39$ & $3(3.1 \%)$ & $6(6 \%)$ & \multirow{4}{*}{$\mathrm{p}=0.347$} \\
\hline $40-49$ & $42(43.75)$ & $48(48.4)$ & \\
\hline $50-59$ & $36(37.5)$ & $24(24.2)$ & \\
\hline $60-69$ & $15(7.6)$ & $21(21.2)$ & \\
\hline \multicolumn{4}{|l|}{ Parity } \\
\hline Nulliparous & $9(9.4)$ & $6(6.1)$ & \multirow{3}{*}{$\mathrm{p}=0.172$} \\
\hline 1 or 2 children & $78(81.2)$ & $66(66.7)$ & \\
\hline$>2$ children & $9(9.4)$ & 27 (27.3) & \\
\hline \multicolumn{4}{|l|}{ Menstrual Status } \\
\hline Premenopausal & $12(12.5)$ & $30(30.3)$ & \multirow{2}{*}{$p=0.081$} \\
\hline Post Menopause & $84(87.5)$ & $69(69.7)$ & \\
\hline \multicolumn{4}{|l|}{ Side } \\
\hline Right & $33(34.4)$ & $48(48.5)$ & \multirow{2}{*}{$\mathrm{p}=0.248$} \\
\hline Left & $66(65.6)$ & $51(51.5)$ & \\
\hline \multicolumn{4}{|l|}{ Histological Type } \\
\hline Infiltrating Ductal & 93 (96.9) & 96 (97) & \multirow{3}{*}{$\mathrm{p}=0.368$} \\
\hline Medullary & 3 & 0 & \\
\hline Cribriform & 0 & 3 & \\
\hline \multicolumn{4}{|l|}{ Tumour Size } \\
\hline Not Known & $21(21.9)$ & $15(15.2)$ & \multirow{4}{*}{$\mathrm{p}=0.890$} \\
\hline$</=2 \mathrm{~cm}$ & $6(6.2)$ & $6(6.1)$ & \\
\hline$>2-5 \mathrm{~cm}$ & $39(40.6)$ & $48(48.5)$ & \\
\hline$>5 \mathrm{~cm}$ & $30(31.2)$ & $30(30.3)$ & \\
\hline \multicolumn{4}{|l|}{ Tumour Grade } \\
\hline Grade 1 & $15(15.6)$ & $12(12.1)$ & \multirow{3}{*}{$\mathrm{p}=0.914$} \\
\hline Grade 2 & $69(71.9)$ & $75(75.8)$ & \\
\hline Grade 3 & $12(12.5)$ & $12(12.1)$ & \\
\hline \multicolumn{4}{|l|}{ Margin } \\
\hline Negative & $84(87.5)$ & 81 (81.8) & \multirow{3}{*}{$P=0.818$} \\
\hline Positive & $6(6.2)$ & $9(9.1)$ & \\
\hline Close & $6(6.2)$ & $9(9.1)$ & \\
\hline \multicolumn{4}{|l|}{ Nodes } \\
\hline Negative & $42(43.8)$ & $48(48.5)$ & \multirow{4}{*}{$P=0.467$} \\
\hline 1-3 Nodes Positive & $27(28.1)$ & $36(36.4)$ & \\
\hline 4-9 Nodes Positive & $18(18.8)$ & $6(6.1)$ & \\
\hline$>10$ Nodes Positive & $9(9.4)$ & $9(9.4)$ & \\
\hline & Table 4 & & \\
\hline
\end{tabular}

\begin{tabular}{|c|c|c|c|}
\hline \multicolumn{4}{|c|}{ Receptor Status } \\
\hline ER & & & \\
\hline Negative & $42(43.8)$ & $57(57.6)$ & \multirow{3}{*}{$p=0.528$} \\
\hline Positive & $45(46.9)$ & $36(36.4)$ & \\
\hline Unknown & $9(9.4)$ & $6(6.1)$ & \\
\hline \multicolumn{4}{|l|}{ PR } \\
\hline Negative & $48(50)$ & $51(51.5)$ & \multirow{3}{*}{$\mathrm{p}=0.882$} \\
\hline Positive & $39(40.6)$ & $42(42.4)$ & \\
\hline Unknown & $9(9.4)$ & $6(6.1)$ & \\
\hline \multicolumn{4}{|l|}{ HER 2 neu } \\
\hline Negative & $48(50)$ & $48(50)$ & \multirow{3}{*}{$\mathrm{p}=0.682$} \\
\hline Positive & $21(21.9)$ & $30(30.3)$ & \\
\hline Unknown & $27(28.1)$ & $21(21.2)$ & \\
\hline $\begin{array}{r}\text { The Table Sho } \\
\text { Populat }\end{array}$ & $\begin{array}{l}\text { Baseline } \\
\text { I Was Com }\end{array}$ & $\begin{array}{l}\text { acteristic } \\
\text { able in Bot }\end{array}$ & $\begin{array}{l}\text { f The Stu } \\
\text { Arms }\end{array}$ \\
\hline
\end{tabular}

\section{Loco-Regional Control}

No patients developed recurrence in chest wall, axilla or supraclavicular fossa. 2 patients developed chest wall nodule during follow up. Both were proven negative for malignancy with repeated fine needle aspiration cytology. They are under follow up.

\section{Toxicity Profile during Radiation Treatment}

\section{Skin Toxicity}

- In Arm A 21.9\% (21) of patients had grade 1 skin toxicity.

- In Arm B 24.2\% (24) had grade 1 skin toxicity.

\begin{tabular}{|c|c|c|c|c|}
\hline & No Skin Toxicity & Grade 1 & Total & p value \\
\hline ARM A & 75 & 21 & 96 & \multirow{2}{*}{0.585} \\
\cline { 1 - 4 } ARM B & 75 & 24 & 99 & \\
\cline { 1 - 4 } Total & 150 & $\mathbf{4 5}$ & 195 & \\
\hline
\end{tabular}

\section{Arm-Wise Distribution}

A total of 45 patients had grade 1 skin toxicity. The occurrence was comparable in both groups ( $p$ value $=0.585$ ). No grade 2,3 or 4 toxicities were recorded. Toxicity occurred during third and fourth week of radiotherapy which subsided by itself.

\section{Lung Toxicity}

- $21.8 \%$ (24) patients in Arm A had grade 1 lung toxicity.

- $\quad$ Grade 1 lung toxicity occurred in $18.1 \%$ (21) of patients in Arm B.

\begin{tabular}{|c|c|c|c|c|}
\hline & No. Lung Toxicity & Grade 1 & Total & p-Value \\
\hline ARM A & 75 & 21 & 96 & \multirow{2}{*}{0.611} \\
\hline ARM B & 81 & 18 & 99 & \\
\hline Total & 156 & 39 & 195 & \\
\cline { 1 - 3 } Distribution of Lung Toxicity in Both Arms Is Shown in \\
This Table \\
\hline
\end{tabular}

\section{Arm-Wise Distribution}

A total of 39 patients had grade 1 lung toxicity during RT. Grade 1 lung toxicity during radiation treatment was comparable in both groups ( $\mathrm{p}$ value $=0.611$ ). No other grades occurred. 7 out of total 15 patients who developed toxicity, had a history of Bronchial Asthma.

\section{Odynophagia}

- Pain during swallowing was the major symptom given by most of the patients.

- $\quad 37.5 \%$ (36) of patients in Arm A had odynophagia.

- $24.2 \%$ (24) of patients developed odynophagia in Arm B.

\begin{tabular}{|c|c|c|c|c|}
\hline & No. Odynophagia & Present & Total & p-Value \\
\hline ARM A & 60 & 36 & 96 & \multirow{2}{*}{0.281} \\
\hline ARM B & 75 & 24 & 99 & \\
\cline { 1 - 4 } Total & 135 & 60 & 195 & \\
\cline { 1 - 3 } This Table Shows Distribution of Odynophagia in Both \\
Arms \\
\hline
\end{tabular}

\section{Arm-Wise Distribution}

20 out of 65 patients had odynophagia mostly during second and third week of radiation. The occurrence of odynophagia was comparable in both groups ( $\mathrm{p}$ value $=0.281$ ) 


\section{Toxicity during Follow Up}

\section{Lymphedema}

- $\quad$ Lymphedema was seen in 9.4\% of patients in Arm A.

- In Arm B 9.4\% had lymphedema.

\begin{tabular}{|c|c|c|c|c|}
\hline & No. Lymphedema & Present & Total & p value \\
\hline ARM A & 87 & 9 & 32 & \multirow{2}{*}{0.968} \\
ARM B & 90 & 9 & 33 & \\
\cline { 1 - 4 } Total & 177 & 18 & 195 & \\
\cline { 1 - 3 } This Table Shows the Distribution of Lymphedema in this \\
\multicolumn{4}{|c|}{ Study Group } \\
\hline
\end{tabular}

18 patients developed lymphedema arm during follow up. This occurred at 9- and 12-months post radiation. The difference between the two arms was not statistically significant $(p$ value $=0.968$ )

\section{Subcutaneous Fibrosis}

- 9 out of 96 patients developed subcutaneous fibrosis in Arm A.

- Subcutaneous fibrosis was seen in 9 out of 99 patients in Arm B.

\begin{tabular}{|c|c|c|c|c|}
\hline & No. Fibrosis & Present & Total & p value \\
\hline ARM A & 87 & 9 & 96 & \multirow{2}{*}{0.968} \\
\cline { 1 - 4 } ARM B & 90 & 3 & 99 & \\
\cline { 1 - 4 } Total & $\mathbf{1 7 7}$ & $\mathbf{1 8}$ & $\mathbf{1 9 5}$ & \\
\cline { 1 - 1 } & This Table Shows the Distribution of Subcutaneous \\
Fibrosis in this Study Group & \\
\hline
\end{tabular}

18 out of total 195 patients developed subcutaneous fibrosis on follow up and was seen at 10 months follow up. No statistically significant difference was seen between the two arms. $(P$ value $=0.968)$

\section{Disease Free Survival}

Disease free survival was also estimated. Disease free survival were calculated from the date of initiation of treatment to the date of disease failure or last follow up. Treatment failures were classified as loco-regional (Chest wall, axilla or supraclavicular fossa) or distant failures (metastasis lung, brain, bones, liver or other viscera)

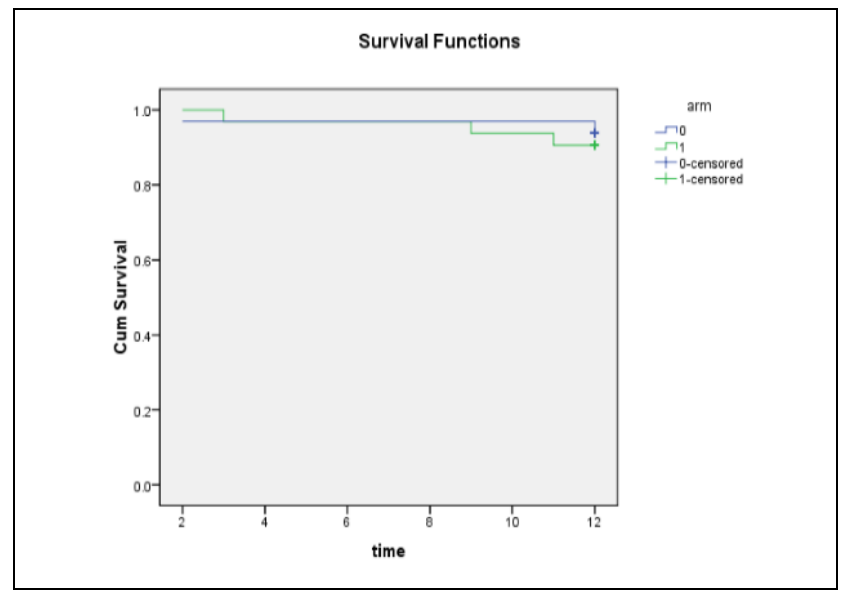

9 patients in Arm B developed brain metastasis. This occurred at second, third and eleventh month of follow-up. 3 patient developed bone and liver metastasis at 9 months. Liver metastasis was also seen in 3 patients at 24 months of follow up. No statistically significant difference in disease free survival was seen between the two arms (Breslow=0.619).

\section{DISCUSSION}

The purpose of this study was to compare two radiation fractionation schedules ( 50 Gy in 25 fractions vs. 40 Gy in 15 fractions) used for chest wall irradiation in the post mastectomy setting in patients with Carcinoma Breast with regard to loco-regional control and toxicity profile. Several studies have shown that two fractionation schedules are equivalent in loco-regional control and cosmetic outcome.

The composition of this study was well matched with respect to age, surgery and other histopathological findings between the two arms. The compliance of patients during this study was one hundred percentage. All patients willingly responded to all questions and came for regular follow up as instructed.

The two groups were matched at baseline. 90 (46.2\%) out of 195 patients in this study belonged to the age group of 40 to 49 years. This age based distribution of patients is similar to the trial conducted by Whelan et al ${ }^{12}$ where majority $(24.7 \%)$ of the patients belonged to the less than 50 year age group. 153 out of 195 patients in this study population were postmenopausal. Only 42 patients were premenopausal, and the difference was found to be statistically significant. This data was comparable to published literature.

114 patients had left sided Breast tumours and the distribution in the study population was statistically significant when compared to right side tumours. But the distribution between the two arms was not significant.

Infiltrating duct carcinoma (NOS) was the histological subtype in $96.9 \%$ (189) patients. In UK START B ${ }^{9}$ trial infiltrating ductal carcinoma accounted for about $77 \%$ of cases. Grade 2 tumours constituted 73.8\% (144/195) of cases in this study compared to $47.4 \%$ of cases in UK START B trial. Subgroup analysis of a study by Whelan et al showed that hypo fractionated RT had an advantage in patients with grade 2 tumours. Grade 2 histology was predominant subtype in our study. Due to the small study population and lack of long term follow up we need further follow up to demonstrate this advantage.

$50.76 \%$ patients had ER negative disease. PR negativity was seen in $50 \%$ and Her 2 neu negativity was seen in $49 \%$ in our study. The study conducted by Whelan et al had $26.1 \%$ of ER negative disease.

All patients underwent Modified Radical Mastectomy as part of surgical management. On follow up for one year no loco-regional recurrences were documented. 3 patients developed chest wall nodule in $2^{\text {nd }}$ year of follow up which were proven positive by repeated fine needle aspiration cytologies and are under follow up 1 patient was in the 50/25 arm and 2 cases in 40/15 arm. The cumulative incidence of loco regional recurrence after 2 years is $1.5 \%$ in 2 years. The cumulative incidence of local recurrence at 10 years was $6.7 \%$ in standard radiation treatment arm compared with $6.2 \%$ in the hypo fractionated group in the trial conducted by Whelan et al. So, our study needs long term follow up to assess recurrence.

The acute toxicities which occurred during RT, recorded in this study were skin toxicity, lung toxicity and odynophagia. Grade 1 skin toxicity occurred in $23 \%$ of patients mainly during third and fourth week of radiation which subsided by 
itself. No other grades of toxicity occurred. The occurrence of grade 1 skin toxicity was found to be equal in both arms where as in a study conducted by E. A. Shaltout, E. Abd El Razek et al,13 in Egypt where 235 women who underwent modified radical mastectomy for treatment of invasive breast cancer were randomized either to receive $50 \mathrm{~Gy}$, in 25 fractions or 40 Gy, in 15 fractions. Acute Skin toxicity occurred in 9 patients $(7.37 \%)$ in group A ( 7 with grade 2 and 2 with grade 3 ), and in 7 cases $(6.2 \%)$ in group B, 6 cases had grade 2 toxicity and 1 case had grade 3 . None of the patients in both groups had grade 4 toxicities. Toxicities were comparable between the two groups In a non-randomized study on 186 patients treated with 44 Gy in 16 fractions over 22 days reported by Olivotto et $\mathrm{al}^{14}$ early toxicity was comparable to their historical patients..

Grade 1 lung toxicity also occurred during RT. The difference in two arms was statistically not significant. This subsided without any intervention

Odynophagia was the most common symptom patients had during RT. But there was no statistically significant difference between two arms. A total of $12 \%$ patients developed lymphedema post radiation treatment in this study over a period of 2 years. This was noted at 9 months and 12 months post radiation treatment. 3 out of 18 patients had a level 3 axillary dissection which may have contributed to development of lymphedema. Two factors consistently shown to impact lymphedema risk are extent of axillary surgery and use of axillary RT. While each of these factors can separately result in risk less than $10 \%$, the additive risk can be as high as $40 \%$ following complete (I to III) axillary dissection and full axillary RT. The frequency varies in different series. Chua BH ${ }^{15}$ reported $9.5 \%$ arm oedema with axillary dissection, $6.1 \%$ with radiation and $31 \%$ when the two modalities were combined $(\mathrm{P}<.001)$. The incidence of lymphedema varies from $25 \%$ to as high as $65 \%$ in various studies. ${ }^{16}$ Long term follow up is warranted to assess the late toxicities of radiation treatment.

No contralateral Breast cancer, secondary malignancy or rib fracture occurred. The development of contralateral breast cancer and secondary malignancy has a long latent period. So we recommend follow-up for longer periods to assess these parameters.

Damage to the brachial plexus is rare but involvement up to $1 \%$ has been described in literature. Match line over dosage could be the cause of this toxicity. The damage to brachial plexus is said to be greater with larger fraction size. But in this study no such problem was encountered. No treatment interruptions occurred.

After 2 year follow up 9 patients developed brain metastasis and received treatment. They were alive during entire follow up period. 3 patients had liver metastasis and bone metastasis and is on chemotherapy. Another patient developed liver metastasis and is on treatment.

\section{CONCLUSION}

The data reported in the present study confirm the feasibility of the hypo fractionated RT with 2.66 Gy per fraction to a total dose of 40 Gy in patients with invasive breast cancer in daily practice. Patients tolerated the treatment well with excellent compliance and nobody stopped the radiotherapy course that lasted 10 days less than that of conventional fractionation.

This hypo fractionated protocol is particularly important in a developing country like India where there is scarcity of resources and financial constraints. By adopting such protocols with equal efficacy and comparable toxicity, we can better utilize available resources and can ensure patient compliance.

In conclusion, after mastectomy for breast cancer, a radiotherapy schedule delivering 40 Gy in 15 fractions over 3 weeks seems to offer local regional tumour control and rates of normal tissue effects are at least as good as the accepted international standard of 50 Gy in 25 fractions over 5 weeks.

\section{REFERENCES}

[1] Koulis TA, Phan T, Olivotto IA. Hypofractionated whole breast radiotherapy: current perspectives. Breast Cancer (Dove Med Press) 2015;7:363-70.

[2] START Trialists' Group, Bentzen SM, Agrawal RK, et al. The UK Standardisation of Breast Radiotherapy (START) Trial A of radiotherapy hypofractionation for treatment of early breast cancer: a randomised trial. The Lancet Oncology 2008;9(4):331-41.

[3] Noone AM Howlader N, Krapcho M, et al. SEER Cancer statistics review, 1975-2015, National Cancer Institute. Bethesda, MD, based on November 2017 SEER data submission, posted to the SEER web site, April 2018. https://seer.cancer.gov/csr/1975_2015/

[4] Gupta S. Breast cancer: Indian experience, data and evidence. South Asian Journal of Cancer 2016;5(3):856.

[5] Gradishar W, Salerno KE. NCCN Guidelines Update: Breast Cancer. Journal of the National Comprehensive Cancer Network JNCCN 2016;14(Suppl 5):641-4.

[6] Early Breast Cancer Trialists' Collaborative Group. Radiotherapy for early breast cancer. The Cochrane Database of Systematic Reviews 2002;(2):CD003647.

[7] Early Breast Cancer Trialists' Collaborative Group (EBCTCG). Effects of chemotherapy and hormonal therapy for early breast cancer on recurrence and 15year survival: an overview of the randomised trials. Lancet 2005;365(9472):1687-717.

[8] Smith BD, Bellon JR, Blitzblau R, et al. Radiation therapy for the whole breast: executive summary of an American Society for Radiation Oncology (ASTRO) evidence-based guideline. Practical Radiation Oncology 2018;8(3):145-52.

[9] START Trialists' Group, Bentzen SM, Agrawal RK, et al. The UK Standardisation of Breast Radiotherapy (START) Trial B of radiotherapy hypofractionation for treatment of early breast cancer: a randomised trial. Lancet 2008;371(9618):1098-107.

[10] Plataniotis G. Hypofractionated radiotherapy in the treatment of early breast cancer. World Journal of Radiology 2010;2(6):197-202.

[11] Pires AM, Segreto RA, Segreto HR. RTOG criteria to evaluate acute skin reaction and its risk factors in patients with breast cancer submitted to radiotherapy. Revista Latino-Americana De Enfermagem 2008;16(5):844-9.

[12] Whelan TJ, Pignol JP, Levine MN, et al. Long-Term results of hypofractionated radiation therapy for breast cancer. New England Journal of Medicine 2010;362(6):513-20. 
[13] Akl FMF, Khater A. Hypofractionated versus conventionally fractionated radiotherapy in postmastectomy breast cancer patients. Journal of Cancer Therapy 2018;9(11):941-54.

[14] Deantonio L, Gambaro G, Beldì D, et al. Hypofractionated radiotherapy after conservative surgery for breast cancer: analysis of acute and late toxicity. Radiation oncology (London, England) 2010;5:112.
[15] Whelan TJ, Olivotto IA, Parulekar WR, et al. Regional nodal irradiation in early-stage breast cancer. The New England Journal of Medicine 2015;373(4):307-16.

[16] Norman SA, Localio AR, Potashnik SL, et al. Lymphedema in breast cancer survivors: incidence, degree, time course, treatment and symptoms. Journal of Clinical Oncology: Official Journal of The American Society of Clinical Oncology 2009;27(3):390-7. 